\title{
Temporal encoding in trace conditioning
}

\author{
ROBERT P. COLE, ROBERT C. BARNET, and RALPH R. MILLER \\ State University of New York, Binghamton, New York
}

\begin{abstract}
Conditioned lick suppression in rats was used to explore the role of timing in trace conditioning. In Experiment 1, two groups of rats were exposed to pairings of a CS (CS1) with a US, under conditions in which the interstimulus interval (ISI) that separated CS1 offset and US onset was either 0 or $5 \mathrm{sec}$. Two additional groups were also exposed to the same CS1 $\rightarrow$ US pairings with either a 0 or a 5-sec ISI, and then received "backward" second-order conditioning in which CS1 was immediately followed by a novel CS2 (i.e., CS1 $\rightarrow$ CS2). A trace conditioning deficit was observed in that the CS1 conditioned with the 5-sec gap supported less excitatory responding than the CS1 conditioned with the 0-sec gap. However, CS2 elicited more conditioned responding in the group trained with the 5-sec CS1-US gap than in the group trained with the 0-sec CS1-US gap. Thus, the CS1-US interval had inverse effects on first- and second-order conditioned responding. Experiment 2 was conducted as a sensory preconditioning analogue to Experiment 1. In Experiment 2, rats received the CS1 $\rightarrow \mathrm{CS} 2$ pairings prior to the CS1 $\rightarrow$ US pairings (in which CS1 was again conditioned with either a 0 or a 5 -sec ISI). Experiment 2 showed a dissociation between first- and second-order conditioned responding similar to that observed in Experiment 1. These outcomes are not compatible with the view that differences in responding to CSs conditioned with different ISIs are mediated exclusively by differences in associative value. The results are discussed in the framework of the temporal coding hypothesis, according to which temporal relationships between events are encoded in elementary associations.
\end{abstract}

In the typical Pavlovian conditioning procedure, an organism is exposed to pairings of a conditioned stimulus (CS) and an unconditioned stimulus (US; Pavlov, 1927). One class of theorizing concerning classical conditioning suggests that the organism forms an association between the CS and US representations that is based on these CS-US pairings. Once established, the CS-US association ordinarily results in behavioral control by the $\mathrm{CS}$ when the CS is presented alone during a test. A fundamental theoretical question concerns how the informational content of these kinds of associations should be characterized (see Rescorla, 1988). Presumably, behavioral control by a CS is observed because the CS informs the organism about an impending US. Indeed, some students of learning have advanced the notion that these associations inform the organism not only that the US is going to occur, but also when in time the US will occur (e.g., Desmond \& Moore, 1988; Logan, 1977; Matzel,

Support for this research was provided by NIMH Grant 33881 and the SUNY-Binghamton Center for Cognitive and Psycholinguistic Sciences. R.C.B. was supported by a Natural Sciences and Engineering Research Council of Canada postgraduate scholarship. We thank Eric Lampinstein, Wendy Packer, and Danielle Scarinci for their assistance in data collection. We also thank Francisco J. Esmoris-Arranz, Lisa M. Gunther, and Hua Yin for comments on earlier versions of the manuscript. Finally, we gratefully acknowledge two anonymous reviewers for pointing out interpretive alternatives for Experiment 1 that led to the design of Experiment 2. Requests for reprints should be addressed to $R$. $R$. Miller, Department of Psychology, SUNY-Binghamton, Binghamton, NY 13902-6000 (e-mail: rmiller@bingvmb.cc.binghamton.edu).

-Accepted by previous editor, Vincent M. LoLordo
Held, \& Miller, 1988; Miller \& Barnet, 1993; Schreurs \& Westbrook, 1982).

The idea that a memory may encode temporal attributes of an experienced event is not new. In the study of human memory, for example, Tulving and Madigan (1970) suggested that memories of discrete events carry information that reflects the points in time at which particular memories have been established. Memories may thus be temporally coded, which could provide organisms with the ability to make judgments about the temporal locations of other events. Jackson (1990; see also Green, 1989) has further suggested that humans may treat temporal relationships between events as information. This information could be stored in memory and, in turn, guide future behavior.

These types of ideas concerning temporal information are captured in a recent view of Pavlovian conditioning which suggests that CS-US associations are composed of more than simple links between event representations. In this framework, the temporal relationships that exist among events during training are encoded as important attributes of the association. This view is called the temporal coding hypothesis (Barnet, Arnold, \& Miller, 1991; Matzel et al., 1988; Miller \& Barnet, 1993). According to this hypothesis, close temporal contiguity between a CS and a US is sufficient for the formation of an association, but a predictive relationship between the CS and US is ordinarily necessary for the behavioral expression of that association.

The assumption of the temporal coding hypothesis, that temporal contiguity is sufficient for associative learning, is challenged by the deficit in conditioned respond- 
ing witnessed in simultaneous conditioning. If temporal contiguity is the primary variable that controls associative acquisition, one might expect that simultaneous conditioning procedures would result in a stronger CS-US association than short-delay forward conditioning procedures, because simultaneous CS-US pairings are an example of perfect contiguity. Consequently, simultaneous CS-US pairings should produce more robust behavioral control by the CS than should short-delay forward CS-US pairings. However, research has repeatedly found that simultaneous conditioning is inferior to forward conditioning (Heth \& Rescorla, 1973; Pavlov, 1927; Smith, Coleman, \& Gormezano, 1969; but see Mahoney \& Ayres, 1976; Rescorla, 1980). Thus, it might be reasonable to assume that the simultaneous conditioning deficit does reflect a deficit in associative acquisition. In this view, simultaneous and forward conditioned associations differ in their associative strength. However, some authors (e.g., Barnet et al., 1991; Matzel et al., 1988) have maintained the position that contiguity is sufficient for associative acquisition and suggest that deficient responding after simultaneous pairings reflects a performance failure rather than a learning failure. This failure in performance may be due to the nature of the responses that experimenters typically assess. Most researchers have examined responses that are anticipatory in nature and thus serve as suitable tests for forward serial learning, but not necessarily so for simultaneous learning (see Barnet et al., 1991, and Matzel et al., 1988, for more detailed discussions).

A divergence in theorizing similar to that concerning simultaneous and forward conditioning may be made concerning trace conditioning. Historically, behavioral research has revealed that the magnitude or frequency of conditioned responding is inversely related to the duration of the trace or interstimulus interval (ISI, defined here as the temporal interval between CS offset and US onset; e.g., Ellison, 1964; Kamin, 1954, 1965; Pavlov, 1927). Thus, for forward paired CSs and USs, a long ISI supports less excitatory responding to the CS than does a short ISI. One reasonable view of this observation is that the ISI affects the strength of the CS-US association in such a way that a longer ISI results in a weaker association than does a shorter ISI. However, without abandoning the concept of associative strength, it is possible that the relationship between ISI and conditioned responding may be understood better if one assumes that a CS-US association conditioned with a long ISI encodes different temporal information than does a CS-US association conditioned with a short ISI. This kind of position is consistent with the temporal coding hypothesis, according to which temporal information is encoded in elementary associations. Differences in behavioral expression between CSs conditioned with short and long ISIs in trace conditioning (or between simultaneous and forward conditioned CSs) may reflect the expression of different temporal information that is encoded in the associations rather than differences in associative strength.
In general, most theories of timing in the animal conditioning literature have focused on temporal information by employing a somewhat different perspective than that of the temporal coding hypothesis. Most notably, Gibbon and Church (1984) have examined the differences between experienced and remembered duration by employing constructs such as internal clocks and counters, whereas Gibbon and Balsam's scalar expectancy theory (1981) concerns itself with the role of timing (e.g., trial spacing) as a determinant of conditioned responding. Although both of these kinds of models provide an important contribution to our understanding of the role of timing in the organization of behavior, neither focally addresses the issue of encoded temporal relationships between paired events or associates. Perhaps the notion of the temporal map proposed by Honig (1981) most closely parallels the temporal coding hypothesis in addressing the encoding of temporal information. Honig's temporal map can be understood most easily through comparison with the more widely known cognitive spatial map, which is an internal representation of the relationships between stimuli in three-dimensional space. The temporal map might best be conceptualized as an internal representation which effectively codes information conveying the temporal relationships between events. Possibly, event durations (e.g., CSs, USs, and ISIs) along with information concerning temporal relationships among events are represented in some temporal field, paralleling the spatial map's representation of location in a spatial field.

In the present series of experiments, we explored this notion of a cognitive temporal map in rats by using a trace conditioning procedure. In Experiment 1, animals were exposed to first-order trace conditioning ( $\mathrm{CS} 1 \rightarrow \mathrm{US}$ ) under conditions in which the duration of the ISI or gap between CS offset and US onset was either 0 or $5 \mathrm{sec}$. On the basis of the existing literature, we anticipated that increases in ISI would degrade the ability of the first-order CS (CS1) to support excitatory conditioned responding. Some animals that received first-order trace conditioning were then exposed to backward second-order conditioning in which the first-order CS1 was forward paired with a novel second-order CS2 (CS1 $\rightarrow$ CS2). Our counterintuitive prediction for Experiment 1 was that animals exposed to the CS $1 \rightarrow$ US training with a 0 -sec ISI would respond in a less robust manner to the second-order CS2 than would animals exposed to the CS1 $\rightarrow$ US training with a 5-sec ISI. This expectation was based on the hypothesis that organisms encode the temporal attributes of stimulus events to which they are exposed (e.g., both order and interval duration) in Pavlovian conditioning and that this temporal information is integrated across separate phases of training. The outcome of such integration is thought of as a composite temporal map which can serve as a basis for generating expectancies about the temporal structure of the environment (e.g., when in time the US is going to occur relative to other stimuli that are present). How such a temporal map could support the specific predictions of the present experiment is consid- 
ered in more detail in the Discussion section. Following training, the degree of conditioned responding was assessed by the abilities of CS1 and CS2 (presented during test sessions) to suppress ongoing drinking behavior in water-deprived rats.

\section{EXPERIMENT 1}

The purpose of Experiment 1 was to evaluate, using a second-order conditioning procedure, the assumption that animals encode the temporal order and duration of events in Pavlovian associations.

\section{Method}

\section{Subjects}

The subjects were 24 male and 24 female experimentally naive, Sprague-Dawley-descended rats. Body-weight ranges were 337$452 \mathrm{~g}$ for males and 218-318 $\mathrm{g}$ for females. Each animal was assigned to one of four groups $(n \mathrm{~s}=12)$ counterbalanced for sex. The animals were individually housed in standard hanging, stainless-steel, wire-mesh cages in a vivarium maintained on a 16:8-h light:dark cycle. Experimental manipulations occurred near the midpoint of the 16-h light cycle. The animals were allowed free access to Purina Laboratory Chow in their home cages. One week prior to the initiation of the study, all animals were progressively deprived of water. By Day 1 of the study, access to water in the home cage was limited to $10 \mathrm{~min}$ per day, which was thereafter provided $18-22 \mathrm{~h}$ prior to any treatment scheduled for the following day. All subjects were handled three times per week, for $30 \mathrm{sec}$, from the time of weaning until the initiation of the study.

\section{Apparatus}

Two types of experimental chambers were used. Chamber A was rectangular in shape and measured $30.30 \times 8.25 \times 12.30 \mathrm{~cm}$ (length $x$ width $\times$ height). The walls and ceiling were constructed of clear Plexiglas, and the floor consisted of stainless steel rods. The rods of the floor measured $0.48 \mathrm{~cm}$ in diameter and were spaced $1.5 \mathrm{~cm}$ apart, center to center. The rods were connected through NE-2 neon bulbs, which allowed constant-current footshock to be delivered by means of a high-voltage $\mathrm{AC}$ circuit in series with a 1.0-M $\Omega$ resistor. Each of six copies of Chamber $A$ was housed in a separate light- and sound-attenuating environmental isolation chest. Chamber A was dimly illuminated by a $2-\mathrm{W}$ (nominal at $120 \mathrm{~V} \mathrm{AC}$ ) bulb driven at $56 \mathrm{~V} \mathrm{AC}$. The bulb was mounted on an inside wall of the environmental chest approximately $30 \mathrm{~cm}$ from the center of the experimental chamber. Background noise, mostly from a ventilation fan, was $74 \mathrm{~dB}(\mathrm{C})$ re SPL.

Chamber $B$ was a $22.30-\mathrm{cm}$-long box in the shape of a vertical truncated V. The chamber was $26.2 \mathrm{~cm}$ high, $21 \mathrm{~cm}$ wide at the top, and narrowed to $5.25 \mathrm{~cm}$ wide at the bottom. The ceiling was constructed of clear Plexiglas, and the walls were constructed of black Plexiglas. The floor consisted of two $25.5-\mathrm{cm}$-long parallel metal plates, each $2 \mathrm{~cm}$ wide and separated by a $1.25-\mathrm{cm}$ gap. A constantcurrent footshock could be delivered through the metal walls and floor of the chamber. Each of six copies of Chamber B was housed in a separate light- and sound-attenuating environmental isolation chest. Chamber B was illuminated by a $7.5-\mathrm{W}$ (nominal at $120 \mathrm{~V}$ $\mathrm{AC}$ ) bulb driven at $56 \mathrm{~V} \mathrm{AC}$. The bulb was mounted on an inside wall of the environmental chest approximately $30 \mathrm{~cm}$ from the center of the experimental chamber. Light entered the experimental chamber primarily by reflection from the roof of the environmental chest. The light intensities in Chamber B roughly matched those in Chamber A, owing to differences in the opaqueness of the walls in Chambers A and B. Background noise, mostly from a ventilation fan, was $74 \mathrm{~dB}(\mathrm{C})$ re SPL.
Chambers A and B could be equipped with a water-filled lick tube. When inserted, the lick tube extended about $1 \mathrm{~cm}$ into a cylindrical drinking recess that was set into one of the narrow Plexiglas walls of the chamber. Each drinking recess was left-right centered on the chamber wall, with its center $1.75 \mathrm{~cm}$ above the floor of the chamber. The recess was $4.5 \mathrm{~cm}$ in diameter and $5.0 \mathrm{~cm}$ deep. An infrared photobeam was projected across the recess approximately $1 \mathrm{~cm}$ in front of the lick tube. In order to drink from the lick tube, subjects had to insert their heads into the recess and thereby break the photobeam. Thus, the duration while subjects were accessing the lick tube could be recorded. All chambers were equipped with two speakers mounted on the interior walls of the environmental chest. Each speaker could deliver a different auditory stimulus, either a click train (6 per second) or a complex tone (3000 and $3200 \mathrm{~Hz}$ ). When presented, the auditory stimuli were $6 \mathrm{~dB}$ above the ambient background noise of $74 \mathrm{~dB}(\mathrm{C})$.

\section{Procedure}

The central features of the procedure are summarized in Table 1. All subjects received trace conditioning with a first-order CS (CS1) and a footshock US during Phase 1 . For half of the subjects (Groups TR-0 and SOC-0), the ISI between CS1 and the US was 0 sec. For the other half of the subjects (Groups TR-5 and SOC-5), the ISI between CS1 and the US was $5.0 \mathrm{sec}$. Following Phase 1, half of the subjects in each ISI condition were tested to assess behavioral control by the trace-conditioned CS1 (Groups TR-0 and TR-5 in Table 1, where TR indicates groups for which the first-order trace CS1 was tested). These subjects were tested at the end of Phase 1 to determine the status of CSI at the initiation of Phase 2 for the remaining animals (i.e., those that would receive secondorder conditioning). The remaining subjects (Groups SOC-0 and SOC-5) were exposed to a second-order conditioning manipulation during Phase 2 , in which a second-order CS2 was backward paired with the first-order CS1 (i.e., $\mathrm{CS} 1 \rightarrow \mathrm{CS} 2$ ). Behavioral control by the second-order CS2 was then assessed in these two remaining groups. Central interest was in the pattern of responding to CS1 and to CS2 as a function of the different ISIs used during Phase 1 trace conditioning.

Acclimation. Acclimation to the chambers was conducted on Days 1 and 2, during which no discrete stimuli were presented. Daily sessions were $60 \mathrm{~min}$, and animals were allowed free access to the water-filled lick tubes. For half of the subjects in each group, Chamber A was designated as the Phase 1 training context (Context Train) and Chamber B was designated as the Phase 2 and testing context (Context Test). For the other half of the animals in each group, these designations were reversed. Subjects were exposed to Context Train on Day 1 and to Context Test on Day 2.

Trace conditioning (Phase 1). Trace conditioning was conducted on Days 3-14 in Context Train. All subjects were exposed to four $\mathrm{CS} \rightarrow$ US pairings during each daily 60 -min session. The trace CS 1

Table 1

Experiment 1: Design Summary

\begin{tabular}{llccc} 
& \multicolumn{4}{c}{ Treatment } \\
\cline { 2 - 5 } Group & \multicolumn{1}{c}{ Phase 1 } & CS1 Test & Phase 2 & CS2 Test \\
\hline TR-0 & CS1 $\rightarrow$ US & CS1? & & \\
TR-5 & CS1 $\longrightarrow$ US & CS1? & & \\
SOC-0 & CS1 $\rightarrow$ US & & CS1 $\rightarrow$ CS2 & CS2? \\
SOC-5 & CS1 $\longrightarrow$ US & & CS1 $\rightarrow$ CS2 & CS2? \\
\hline
\end{tabular}

Note-All groups received trace conditioning with CS1 during Phase 1. The ISI between CS1 and the US during Phase 1 was either 0 sec (denoted by short arrow, Groups TR- 0 and SOC- 0 ) or $5 \mathrm{sec}$ (denoted by long arrow, Groups TR-5 and SOC-5). Following Phase 1 trace conditioning, Groups TR-0 and TR-5 were tested for suppression to CS1. Groups SOC-0 and SOC-5 received second-order conditioning in Phase 2 and were then tested for suppression to CS2 
was a complex tone of 5-sec duration. The US was a $0.5-\mathrm{sec}, 0.8-\mathrm{mA}$ footshock. For Groups TR-0 and SOC-0, the US was delivered immediately upon offset of CS1. For Groups TR-5 and SOC-5, the US was delivered $5 \mathrm{sec}$ after CS offset. One of two different schedules of training was used in each session, pseudorandomly selected throughout Phase 1 . In one schedule, the trials began $8,28,37$, and $55 \mathrm{~min}$ into the session. In the other schedule, trials began 10, 20, 37 , and $50 \mathrm{~min}$ into the session. The lick tubes were not available during trace conditioning.

Reacclimation. Groups TR-0 and TR-5 received daily $60-\mathrm{min}$ sessions in Context Test on Days 15 and 16 during which no discrete stimuli were presented. The water-filled lick tubes were available. The purpose of the reacclimation sessions was to reestablish a stable rate of drinking from the lick tubes prior to testing of CS1.

Trace testing. On Day 17, Groups TR-0 and TR-5 were tested in Context Test for conditioned suppression of ongoing licking in the presence of CS1. Our rationale for testing these subjects was to assess the subjects' sensitivity to the trace manipulation without contamination from further training. Following the completion of the first 5 cumulative seconds of licking upon placement in the chambers (measured by cumulative time during which the infrared photobeam was broken), CS1 was presented. Pre-CS1 latencies to complete this first 5 cumulative seconds of licking and latencies to complete an additional 5 cumulative seconds of licking in the presence of CS1 were recorded. A ceiling of $10 \mathrm{~min}$ was imposed on latencies to lick for 5 cumulative seconds in the presence of CS1. Any animal that took longer than $60 \mathrm{sec}$ to complete its first 5 cumulative seconds of drinking upon placement in the chamber during test sessions for CS1 and CS2 (i.e., prior to onset of CS1 or CS2) was eliminated from the study. One animal from Group TR- 0 met this pre-CS exclusion criterion and was eliminated from the experiment.

Second-order conditioning (Phase 2). On Day 15, Groups SOC0 and SOC-5 were exposed to a second-order conditioning manipulation in an associatively neutral context (Context Test). Context Test, in which no USs had ever been presented, was used for secondorder conditioning in order to decrease the possibility that contextUS associations would contribute to any second-order conditioning to CS2 that might emerge (e.g., Marlin, 1983). During this 60-min session, subjects were exposed to four backward pairings of $\mathrm{CS} 2$ with $\mathrm{CS} 1$ (i.e., $\mathrm{CS} 1 \rightarrow \mathrm{CS} 2$ ). $\mathrm{CS} 2$ was a click train with a 5 sec duration and was presented immediately following the offset of CS1. The trials began 10, 20,37, and 50 min into the session. Lick tubes were not available during second-order conditioning.

Reacclimation. On Days 16-17, Groups SOC-0 and SOC-5 received reacclimation sessions in Context Test identical to those received by Groups TR- 0 and TR- 5 following trace conditioning.
Second-order testing. On Day 18, Groups SOC-0 and SOC-5 were tested for conditioned suppression of ongoing licking in the presence of CS2 in Context Test. The testing procedure was identical to that administered to Groups TR-0 and TR-5, except that CS2 was presented as the test stimulus. One animal from Group SOC-5 met the pre-CS exclusion criterion and was eliminated from the study.

All suppression data were converted into log latencies (base 10) in order to permit the use of parametric statistics. An alpha level of .05 was adopted for tests of statistical significance.

\section{Results and Discussion}

The primary observation emerging from this experiment was that the pattern of responding across the different trace intervals was dissociated in the tests measuring responding to the first-order CS (CS1) and the second-order CS (CS2).

\section{Trace Conditioning Data}

The left side of Figure 1 illustrates suppression means to CS1 for Groups TR-0 and TR-5. A one-way ANOVA revealed an effect of trace interval $[F(1,21)=8.23, p<.01]$. As can be seen in Figure 1, Group TR-5 exhibited less suppression to CS1 than did Group TR-0. This indicates that the animals were sensitive to the different trace values. As ISI increased, suppression latencies to CS1 decreased. An analysis of pre-CS1 latencies failed to reveal differences between Groups TR-0 and TR-5 $(F<1)$.

\section{Second-Order Data}

The right side of Figure 1 depicts suppression means to CS2 for Groups SOC-0 and SOC-5. A one-way ANOVA used to assess differences in mean suppression latencies between Groups SOC- 0 and SOC- 5 revealed an effect of the Phase 1 trace interval $[F(1,21)=20.88, p<.001]$. As inspection of Figure 1 reveals, Group SOC-5 exhibited greater suppression to CS2 than did Group SOC-0. As the Phase 1 ISI increased, suppression latencies to CS2 increased. This finding is in contrast to the pattern of responding that was observed to CS1 across the different trace values. An analysis of pre-CS2 latencies

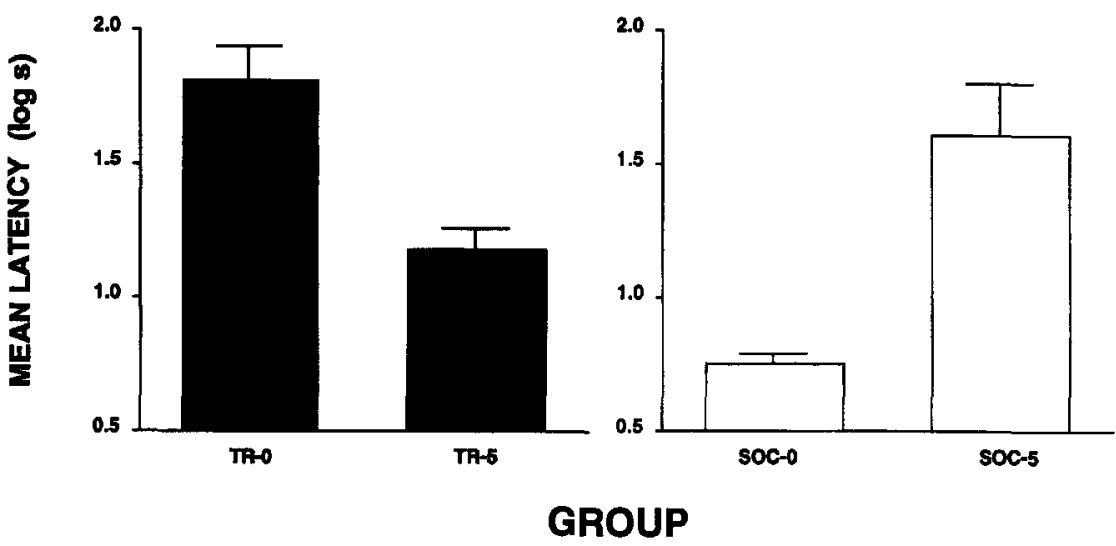

Figure 1. Mean latency to lick for 5 cumulative seconds in presence of CS1 (solid bars) or CS2 (open bars), as a function of treatment group in Experiment 1. Brackets indicate standard errors. 
failed to reveal differences between Groups SOC-0 and SOC-5 $(F<1)$.

It is interesting that the effect of variation in the CSI-US interval on responding to the second-order CS2 in the present experiment is in contrast to observations made by Kehoe, Feyer, and Moses (1981, Experiment 3). Those authors, using the rabbit's nictitating membrane response, also investigated the effect of variation in the ISI between CS1 and a US on excitatory responding to a second-order CS2. Kehoe et al., however, used a more conventional forward second-order conditioning procedure in which $\mathrm{CS} 2$ preceded $\mathrm{CS} 1(\mathrm{CS} 2 \rightarrow \mathrm{CS} 1)$. In the present experiment, the effects of variation in the CS1-US interval on responding to CS1 and CS2 were dissociated. In the Kehoe et al. report, the response potential to CS2 was predictable from and parallel to the response potential of CS1. In other words, varying the CS1-US interval in that experiment had the same effect on responding to CS2 as it did on responding to CS1. One implication of this observation is that, in forward second-order conditioning, the associative value of CS1 is directly related to the acquisition of second-order conditioning to CS2. Although there was clearly a systematic relationship between the response potentials of CS1 and CS2 in the present experiment, that relationship is not readily explained by differences in the associative value of CSI (at least as conventionally viewed with increasing ISI resulting in decreased associative strength).

The CS1 data are consistent with the previously reported data concerning the trace conditioning deficit (e.g., Ellison, 1964; Kamin, 1954, 1965; Pavlov, 1927). That is, an inverse relationship was seen between the length of the CS1-US ISI and conditioned responding to CS1. Thus, the low level of suppression to CS1 in Group TR-5 may have occurred because the CS1-US association in this group was weak, relative to the strength of the CS1-US association in Group TR-0. This conventional view concerning the trace conditioning deficit cannot easily explain the present data. Differences in responding to CS1 in Groups TR- 0 and TR- 5 might be explained by assuming that CS1 in these groups differed in associative strength (Pavlov, 1927). However, this simple view makes predictions of a different nature concerning responding to CS2 than those based on the temporal coding hypothesis. Specifically, if the associative value of CS1 in Group TR-5 was relatively weak, then any second-order conditioning based on pairings with CS1 should also have been relatively weak. The emergent prediction from this view is that responding to CS2 in Group SOC-5 should have been weak relative to responding to CS2 in Group SOC- 0 . Such a prediction was not supported in the present experiment. Rather, responding to CS2 by Group SOC-5 (which was based on the presumably "weak" CS1) was superior to the degree of responding to CS2 exhibited by Group SOC-0 (which was based on the presumably "strong" CS1). This dissociation in responding to $\mathrm{CS} 2$ relative to $\mathrm{CS} 1$ suggests that variations in the associative value of CS1 may not adequately explain both the observed difference in responding to $\mathrm{CS} 1$ and the observed difference in responding to CS2.

An alternative interpretation of these data is provided by the temporal coding hypothesis (Barnet et al., 1991; Matzel et al. 1988). Recall that the temporal coding hypothesis assumes that elementary associations are composed not only of event representations, but also of the temporal relationships that prevail among the events during training. The temporal encoding view of the secondorder CS2 data in the present experiment is that order and interval information from Phase 1 (e.g., CS1-US order, CS1 duration, and the ISI associated with CS1) was encoded, and integrated or superimposed with the order and interval information from Phase 2 (e.g., that CS1 predicts CS2, and CS2 duration). The idea of the superimposition of representations in memory, or memory blending, is consistent with recent theorizing about episodic memory in human subjects. Metcalfe (1990), for example, has suggested that a given memory can be thought of as a composite or blending of different learning episodes. This view is compatible with the notion of a temporal map (Honig, 1981) in that the temporal map could be thought of as a memory representation that codes superimposed or blended temporal attributes from different learning experiences. The diagrams in Figure 2 illustrate one possibility for the nature of the temporal information that such a temporal map could provide, and how differences in that temporal information might serve as a basis for understanding the second-order CS2 (as well as the CS1) data from the present experiment.

The possibility considered here is that Phase 1 conditioning resulted in CS1-US associations that encode the temporal location of the US. As the top portion of Figure 2 suggests, animals trained in Phase 1 with a $0-\mathrm{sec}$ delay learned to expect the US immediately at CS1 offset (i.e., Group TR-0). Animals trained with a 5-sec delay learned to expect the US $5 \mathrm{sec}$ following $\mathrm{CS} 1$ offset (i.e., Group TR-5). Thus, differences in behavior controlled by CS1 may be attributed to differences in the nature of the US expectancy activated by CS1. Because CS1 predicted a more immediate US in Group TR-0, excitatory responding in this group was superior. In this view, Phase 2 training resulted in a memory representation that codes the order (and possibly duration) of the Phase 2 events - namely, that CS1 predicts CS2. The bottom portion of Figure 2 illustrates this possibility. The idea that temporal information from Phase 1 (i.e., the CS1-US interval) was integrated with temporal information from Phase 2 (i.e., the $\mathrm{CS} 1 \rightarrow \mathrm{CS} 2$ temporal relationship) is illustrated in the bottom portion of Figure 2 by placement of the solid black box depicting memory for the US. Note that the temporal relationship between CS1 and the US, learned in Phase 1, has been preserved in the superimposed or blended memory representation resulting from Phase 2. ${ }^{1}$ The consequence of this preservation in the composite temporal map is that the temporal location of the US, relative to the stored location of CS2, differs between Groups SOC-0 and SOC-5. As sug- 
PRESUMED TEMPORAL AND EVENT REPRESENTATIONS

GROUP PRESUMED PHASE 1 MEMORY REPRESENTATION

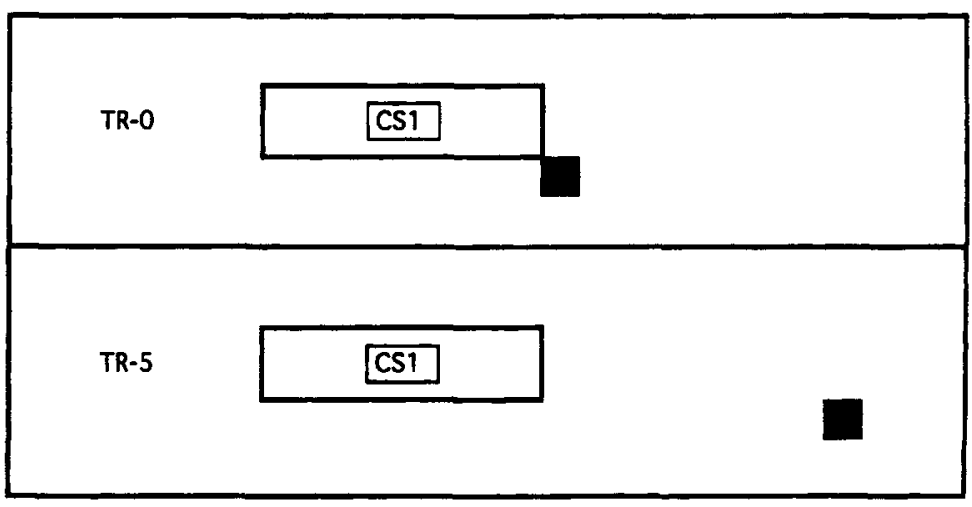

GROUP PRESUMED SUPEAIMPOSED MEMORY REPRESENTATION

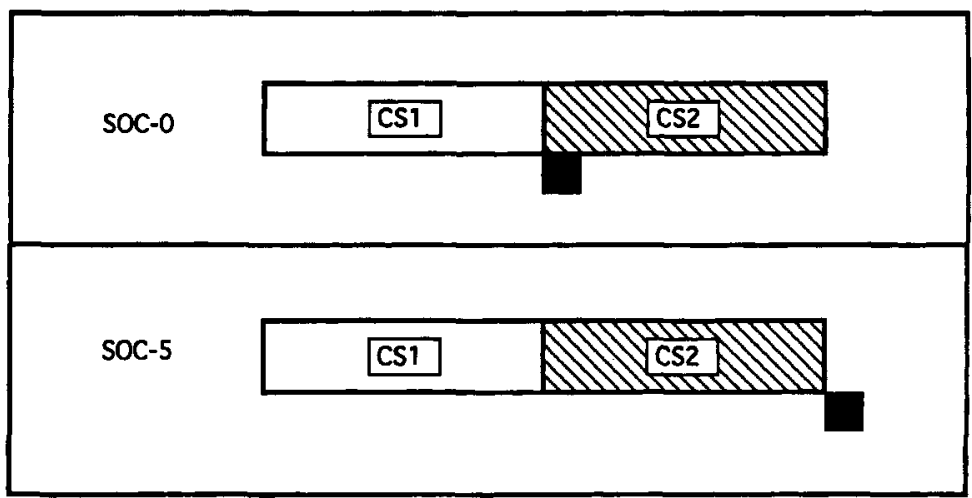

Figure 2. Presumed temporal and event representations that underlie suppression to CS1 (top panel) and CS2 (bottom panel). The black square represents the unconditioned stimulus.

gested by Figure 2, the difference between information concerning CS 2 encoded by Groups SOC- 0 and SOC-5 is in the nature of the temporal expectancy of the US. Only in Group SOC-5 does CS2 bear a predicative relationship to the (memory for the) US. It is this predicative relationship between CS2 and the US in the composite temporal map that is presumed to support the robust excitatory responding to CS2 observed in Group SOC-5.

Similarly, the absence of a predictive relationship between memories for CS2 and the US in the composite temporal map of Group SOC-0 may explain why Group SOC-0 did not exhibit strong excitatory responding to CS2. Thus, presentation of CS2 during the test activated an expectancy of the US, and the temporal nature of that expectancy differed between Groups SOC-0 and SOC-5. This account is consistent with the assumption of the temporal coding hypothesis that a predictive relationship between events (e.g., CS1 and the US) or memories for events (e.g., CS2 and the US) is a necessary condition for the expression of associative learning. One important, although less obvious, contribution of the present analysis is that it focuses theoretical attention on the integration of (temporal) information across different learning episodes. This is an important contribution, because the nature and role of integration of information in animal memory has often been overlooked (Medin \& Dewey, 1986, p. 183).

As previously suggested, an explanation of the present findings based on the associative value of CS1 being weaker in Group SOC-5 than in Group SOC-0 cannot, by simple mediated excitation, explain the differences in responding to CS2 that were observed. However, there are viable explanations of the observed differences in responding to CS2 that are based on variations in the associative strength of CS1. Consider two such explanations that do not demand assumptions of temporal encoding.

One alternative interpretation is that CS2 acquired inhibitory strength during Phase 2 training and that this inhibitory strength was greater for Group SOC- 0 than for Group SOC-5. This argument is predicated on the as- 
sumption of some theoretical models of conditioned inhibition that inhibition to a target $C S$ is acquired when the CS is nonreinforced in the presence of a strong excitor (e.g., Rescorla \& Wagner, 1972). In the present experiment, CS2 was nonreinforced in the presence of a previously reinforced stimulus, CS1. Moreover, the excitatory associative value of CS1 could reasonably be expected to be greater in Group SOC-0 than in Group SOC-5 because during Phase 1 CS1 had enjoyed superior temporal contiguity with the US in Group SOC-0 (and had accordingly supported robust excitatory control in Group TR-0; see the left panel of Figure 1). In this analysis, CS2 was nonreinforced in the presence of a strong excitor in Group SOC-0 (a condition especially favorable for the acquisition of inhibition), but was nonreinforced in the presence of a weak excitor in Group SOC-5 (a condition less favorable for the acquisition of inhibition). Thus, excitatory control by CS2 may have been stronger in Group SOC-5 than in Group SOC-0, not because CS2 was more excitatory in Group SOC-5, but rather because CS2 was more inhibitory in Group SOC $-0 .^{2}$

A second alternative interpretation is that CS2 supported less excitatory responding in Group SOC-0 than in Group SOC-5 because CS2 was less well learned about in Group SOC-0. In this view, as in the preceding inhibition account, the excitatory associative value of CS1 is presumed to be greater in Group SOC-0 than in Group SOC-5. Recall that second-order conditioning with CS2 consisted of serial CS1 $\rightarrow \mathrm{CS} 2$ pairings in all animals. Therefore, CS2 was immediately preceded by a stronger excitor in Group SOC-0 than in Group SOC-5. The central possibility here is that CS1 distracted animals from processing CS2 more in Group SOC-0 (for which CS1 was a strong excitor and therefore able to promote considerable interference or distraction) than in Group SOC-5 (for which CS1 was a weak excitor and therefore less able to promote distraction or interference). In this interference or distraction view, to the extent that CS2 was less well processed in Group SOC-0, CS2 should have been less likely to enter into associations of any kind with other stimuli and therefore should have been less capable of supporting excitatory responding.

Thus, the conditioned inhibition and interference views offer alternative explanations of the findings from Experiment 1 to that provided by the temporal coding hypothesis. However, note that the conditioned inhibition account and the interference view both demand that the excitatory value of CS1 be different in Group SOC-0 and Group SOC-5 at the time that CS2 was paired with CS1. That is, differences in the excitatory value of CS1 in Groups SOC-0 and SOC-5 must have been present at the time of CS1 $\rightarrow$ CS2 pairings in order for CS1 to differentially influence the nature or course of learning about CS2 in Groups SOC-0 and SOC-5. Experiment 2 was designed to evaluate the conditioned inhibition and interference accounts by eliminating the contribution of such differences in the associative value of CSI at the time of the $\mathrm{CS} 1 \rightarrow \mathrm{CS} 2$ pairings.

\section{EXPERIMENT 2}

Experiment 2 was conducted as a sensory preconditioning analogue to the second-order conditioning procedure that was used in Experiment 1. In Experiment 1, Phase 1 training with CS1 (CS $1 \rightarrow$ US) preceded Phase 2 training with CS1 and CS2 (CS1 $\rightarrow$ CS2). Sensory preconditioning is procedurally similar to second-order conditioning except that the two phases of training are reversed. Thus, in Experiment 2, all CS1 $\rightarrow \mathrm{CS} 2$ training preceded CS $1 \rightarrow$ US training. The important advantage of the sensory preconditioning procedure in the present case was that it allowed training with CS2 to occur at a time during which the associative value of CS1 was the same for all animals. If differences in the associative value of CS1 at the time of training with $\mathrm{CS} 2$ were important determinants of the pattern of responding to CS2 in Groups SOC-0 and SOC-5 from Experiment 1, eliminating the differential experience with $\mathrm{CS} 1$ at the time of CS1 $\rightarrow \mathrm{CS} 2$ pairings in Experiment 2 should eliminate the between-group differences in responding to CS2 . However, if differences in responding to CS2 were controlled by differences in temporal expectancies for the US as suggested by the composite temporal map of Figure 2, the central predictions for responding to CS2 would be unchanged for Experiments 1 and 2. That is, changing the order in which the training phases are presented does not change the predictions of the temporal coding hypothesis.

\section{Method}

\section{Subjects and Apparatus}

Twelve male and 12 female experimentally naive SpragueDawley-descended rats obtained from our own breeding colony served as subjects. Body weights ranged from 201 to $460 \mathrm{~g}$ for males and from 174 to $320 \mathrm{~g}$ for females. Subjects were assigned to one of two different treatment groups $(n=12)$ counterbalanced for sex. Animal care, maintenance, and the apparatus were the same as in Experiment 1.

\section{Procedure}

The central features of Experiment 2 are summarized in Table 2. In Phase 1, all subjects were exposed to $\mathrm{CS} 1 \rightarrow \mathrm{CS} 2$ pairings. Following Phase 1 training, some subjects received $\mathrm{CS} 1 \rightarrow$ US pairings with a 0 -sec ISI between CS1 and the US (Group SPC-0), while others received $\mathrm{CS} 1 \rightarrow$ US pairings with a 5 -sec ISI between CS1 and the US (Group SPC-5). Both groups were then tested for suppression to CS2 and CS1. Independent groups of animals were

Table 2

Experiment 2: Design Summary

\begin{tabular}{lcccc}
\hline & \multicolumn{4}{c}{ Treatment } \\
\cline { 2 - 5 } Group & Phase 1 & \multicolumn{1}{c}{ Phase 2 } & CS2 Test & CS1 Test \\
\hline SPC-0 & CS1 $\rightarrow$ CS2 & CS1 $\rightarrow$ US & CS2? & CS1? \\
SPC-5 & CS1 $\rightarrow$ CS2 & CS1 $\longrightarrow$ US & CS2? & CS1? \\
\hline
\end{tabular}

Note-All groups received sensory preconditioning with CS1 and CS2 in Phase 1. In Phase 2, all groups received trace conditioning with CS1. The ISI between CS 1 and the US during Phase 2 was either 0 sec (denoted by short arrow, Group SPC-0) or $5 \mathrm{sec}$ (denoted by long arrow, Group SPC-5). 
not used for tests with only CS1 as had been done in Experiment 1 (TR groups). In Experiment 1, TR groups served to demonstrate that responding to CS1 was sensitive to the trace conditioning manipulation in tests that were uncontaminated by other (i.e., subsequent) training. Use of the sensory preconditioning procedure, in which some training precedes the CS1 $\rightarrow$ US pairings, precluded this possibility. Thus, there was little value of TR groups for testing with CS1 in Experiment 2. Procedural details and stimuli were identical to that of Experiment 1 except where otherwise noted.

Acclimation. Acclimation to the chambers was conducted on Days 1 and 2, during which no discrete stimuli were presented. For half of the subjects in each group, Chamber A was designated as the Phase 1 training context (Context Train) and Chamber B was designated as the Phase 2 and testing context (Context Test). For the other half of the animals in each group, these designations were reversed. Subjects were exposed to Context Train on Day 1 and to Context Test on Day 2. Daily sessions were $60 \mathrm{~min}$, and animals were allowed free access to the water-filled lick tubes.

Sensory preconditioning (Phase 1). Following acclimation, sensory preconditioning was conducted on Days 3-5 in Context Train. All subjects were exposed to four $\mathrm{CS} 1 \rightarrow \mathrm{CS} 2$ pairings during each daily 60 -min session. $\mathrm{CS} 1 \rightarrow \mathrm{CS} 2$ training was conducted for 3 days, rather than 1 day as in Experiment 1 , because pilot studies indicated that one day of $\mathrm{CS} 1 \rightarrow \mathrm{CS} 2$ training failed to support appreciable responding to $\mathrm{CS} 2$ during subsequent testing.

Trace conditioning (Phase 2). Following acclimation, trace conditioning was conducted on Days 6 and 7 in Context Train. All subjects were exposed to four $\mathrm{CS} 1 \rightarrow$ US pairings during each daily 60 min session. For Group SPC-0, the US was delivered immediately upon offset of CS1. For Group SPC-5, the US was delivered $5 \mathrm{sec}$ after CS offset.

Reacclimation. Following trace conditioning, all subjects received daily 60-min sessions in Context Test on Days 8-10 during which no discrete stimuli were presented. The water-filled lick tubes were available. The purpose of the reacclimation sessions was to reestablish a stable rate of drinking from the lick tubes prior to testing.

Second-order testing. On Day 11 , all subjects were tested in Context Test for conditioned suppression of ongoing licking in the presence of CS2. Following completion of the first 5 cumulative seconds of licking, CS2 was presented. Pre-CS2 latencies to complete this first 5 cumulative seconds of licking and latencies to complete an additional 5 cumulative seconds of licking in the presence of CS2 were recorded. A ceiling of $10 \mathrm{~min}$ was imposed on latencies to lick (for 5 cumulative seconds) in the presence of CS2. Any animal that took longer than 60 seconds to complete its first $5 \mathrm{cu}$ mulative seconds of drinking upon placement in the chamber (i.e., prior to onset of CS2) was eliminated from the study. No animals met this criterion.
First-order testing. On Day 12, all subjects were tested for conditioned suppression of ongoing licking in the presence of CS1 in Context Test. The testing procedure was identical to the testing administered on Day 11, except that CS1 was presented instead of $\mathrm{CS} 2$. The elimination criterion based on pre-CS behavior was identical to that of second-order testing, and again no animals met this criterion.

\section{Results and Discussion}

The primary observation emerging from Experiment 2 was that the pattern of responding across the different trace intervals was dissociated in the tests of responding to the first-order CS (CS1) and the second-order CS (CS2). This dissociation is similar to that previously observed in Experiment 1.

\section{Trace Conditioning Data}

The left side of Figure 3 illustrates suppression means to the first-order CS (CS1) for Groups SPC-0 and SPC-5. As can be seen in Figure 3, Group SPC-0 exhibited greater suppression to CS1 than did Group SPC-5, indicating that the animals were sensitive to the different trace values $[F(1,22)=28.51, p<.001]$. As ISI increased, suppression latencies to CS1 decreased. This observation is consistent with the results of Experiment 1 . As anticipated, analysis of pre-CS data failed to reveal differences in preCS licking between Groups SPC-0 and SPC-5 $(F<1)$.

\section{Second-Order Data}

The right side of Figure 3 depicts suppression means to CS2 for Groups SPC-0 and SPC-5. As inspection of Figure 3 reveals, Group SPC-5 exhibited greater suppression to $\mathrm{CS} 2$ than did Group SPC-0 $[F(1,22)=36.79, p<.001]$. As ISI increased, suppression latencies to CS2 increased. This finding is in contrast to the pattern of responding that was observed to CS1 across the two trace values and is again consistent with the results of Experiment 1. Again as expected, an analysis of pre-CS2 latencies failed to reveal differences between Groups SPC-0 and SPC-5 $(F<1)$.

As detailed in the discussion of Experiment 1, the temporal coding view was not the only plausible interpretation that could explain the results from Experiment 1. Explanations based on conditioned inhibition and inter-

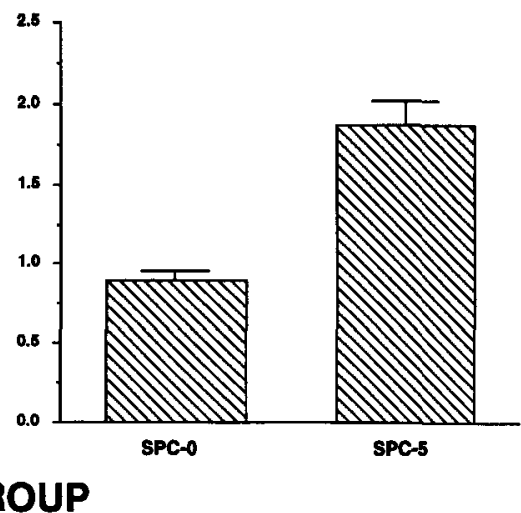

Figure 3. Mean latency to lick for 5 cumulative seconds in presence of CS1 (solid bars) or CS2 (hatched bars), as a function of treatment group in Experiment 2. Brackets indicate standard errors. 
ference are also compatible with that data. However, both of these views rely on differential CS1-US experience prior to CS2 training. Importantly, the critical differential experience with CS1 before training with CS2 was eliminated in the sensory preconditioning procedure of Experiment 2. Despite this change, the pattern of responding to CS2 and CS1 in Experiment 2 was similar to that of Experiment 1. Therefore, the conditioned inhibition and interference alternatives do not offer a cohesive explanation of the findings of the present experiments. The outcomes of both experiments, however, may be understood in the framework of the temporal coding hypothesis. In this framework, the dissociation between first- and second-order responding is understood by assuming that animals to some extent encoded the temporal order and duration of the events to which they were exposed. Moreover, this view suggests that the animals integrated the temporal memories across separate phases of training.

Beyond the implications that these data have for the coding of temporal information in animal memory, the data strongly encourage a distinction between learning and performance in trace conditioning. The superior responding to CS2 by subjects in Groups SOC-5 (relative to Group SOC-0 in Experiment 1) and SPC-5 (relative to Group SPC-0 in Experiment 2), as compared with the inferior responding to CS1 by subjects in Groups TR-5 (relative to Group TR-0 in Experiment 1) and SPC-0 (relative to Group SPC-5 in Experiment 2), indicates that subjects in Groups TR-5 in Experiment 1 and Group SPC-5 in Experiment 2 had access to more information about CS1 than was apparent in their responding to that stimulus. That is, the deficient responding to CS1 in Groups TR-5 and SPC-5 was not the result of a failure to acquire a strong CS1-US association. Indeed, it was the presence of a robust CS1-US association in Groups SOC-5 and SPC-5 that is presumed to have resulted in a strong (and predictive) CS2-US association in these groups. Differences in behavior controlled by CS1 in Groups TR-0 and TR-5 (in Experiment 1) and SPC-0 and SPC-5 (in Experiment 2) could well reflect the expression of different temporal information that was encoded in the CS1-US associations, rather than differences in the strength of the CS1-US associations. Thus, the present view does not demand that the associative strength of CS1 differ between groups conditioned with 0 -sec versus 5-sec ISIs (although associative strength would presumably decrease with longer trace intervals). ${ }^{3}$

Similar arguments have been made by Barnet et al. (1991) and Matzel et al. (1988) concerning simultaneous and forward conditioning. According to those authors, the inferior responding that often emerges in simultaneous relative to forward conditioning does not necessitate the interpretation that simultaneous conditioning procedures produce weaker learning. In summary, the dissociation between first-order and second-order responding in the present experiments suggests some performance failure in trace conditioning rather than an impairment of acquisition when a CS and US are paired with a long ISI.
Resolution of these kinds of issues may be facilitated by a theoretical analysis of elementary associations in which timing is viewed as an attribute of the association and not only as a determinant of associative strength.

\section{REFERENCES}

Barnet, R. C., ARnold, H. M., \& Miller, R. R. (1991). Simultaneous conditioning demonstrated in second-order conditioning: Evidence for similar associative structure in forward and simultaneous conditioning. Learning \& Motivation, 22, 253-268.

Desmond, J. E., \& Moore, J. W. (1988). Adaptive timing in neural networks: The conditioned response. Biological Cybernetics, 58, 405415.

Ellison, G. D. (1964). Differential salivary conditioning to traces. Journal of Comparative \& Physiological Psychology, 57, 373-380.

GiBbon, J., \& BalSaM, P. D. (1981). Spreading association in time. In C. M. Locurto, H. S. Terrace, \& J. Gibbon (Eds.), Autoshaping and conditioning theory (pp. 219-253). New York: Academic Press.

GibBon, J., \& CHURCH, R. M. (1984). Sources of variance in an information processing theory of timing. In H. L. Roitblat, T. G. Bever, \& H. S. Terrace (Eds.), Animal cognition (pp. 465-488). Hillsdale, $\mathrm{NJ}$ : Erlbaum.

GREEN, R. L. (1989). Spacing effects in memory: Evidence for a twoprocess account. Journal of Experimental Psychology: Learning, Memory, \& Cognition, 15, 371-377.

HeTH, C. D., \& Rescorla, R. A. (1973). Simultaneous and backward fear conditioning in the rat. Journal of Comparative \& Physiological Psychology, 82, 434-443.

HoNiG, W. K. (1981). Working memory and the temporal map. In N. E. Spear \& R. R. Miller (Eds.), Information processing in animals: Memory mechanisms (pp. 167-197). Hillsdale, NJ: Erlbaum.

JACKSON, J. L. (1990). A cognitive approach to temporal information processing. In R. A. Block (Ed.), Cognitive models of psychological time (pp. 153-180). Hillsdale, NJ: Erlbaum.

KamiN, L. J. (1954). Traumatic avoidance learning: The effects of CS-US interval with a trace conditioning procedure. Journal of Comparative \& Physiological Psychology, 47, 65-72.

Kamin, L. J. (1965). Temporal and intensity characteristics of the conditioned stimulus. In W. F. Prokasy (Ed.), Classical conditioning (pp. 118-147). New York: Appleton-Century-Crofts.

KeHOE, E. J., Feyer, A. M., \& Moses, J. L. (1981). Second-order conditioning of the rabbit's nictitating membrane response as a function of the CS2-CS1 and CS1-US intervals. Animal Learning \& Behavior, 9, 304-315.

LOGAN, F. A. (1977). Hybrid theory of classical conditioning. In G. H. Bower (Ed.), The psychology of learning and motivation (Vol. 11, pp. 203-243). New York: Academic Press.

Mahoney, W. J., \& Ayres, J. J. B. (1976). One-trial simultaneous and backward fear conditioning as reflected in conditioned suppression of licking in rats. Animal Learning \& Behavior, 4, 357-362.

MARLIN, N. A. (1983). Second-order conditioning using a contextual stimulus as $\mathrm{S}_{1}$. Animal Learning \& Behavior, 11, 290-294.

Matzel, L. D., Held, F. P., \& MilleR, R. R. (1988). Information and the expression of simultaneous and backward associations: Implications for contiguity theory. Learning \& Motivation, 19, 317-344.

Medin, D. L., \& DewEY, G. I. (1986). Memory theories: Past, present, and projected. In D. F. Kendrick, M. E. Rilling, \& M. R. Denny (Eds.), Theories of animal memory (pp. 173-196). Hillsdale, NJ: Erlbaum.

MetCalfe, J. (1990). Composite holographic associative recall model (CHARM) and blended memories in eyewitness testimony. Journal of Experimental Psychology: General, 119, 145-160.

Miller, R. R., \& BarNet, R. C. (1993). The role of time in elementary associations. Current Directions in Psychological Science, 2, 106-111.

Pavlov, I. P. (1927). Conditioned reflexes (G. V. Anrep, Trans.). London: Oxford University Press.

RESCORLA, R. A. (1980). Simultaneous and successive associations in sensory preconditioning. Journal of Experimental Psychology: Animal Behavior Processes, 6, 207-216. 
ResCoRla, R. A. (1988). Pavlovian conditioning: It's not what you think it is. American Psychologist, 43, 151-160.

RESCORLA, R. A., \& WAGNER, A. R. ( 1972). A theory of Pavlovian conditioning: Variations in the effectiveness of reinforcement and nonreinforcement. In A. H. Black \& W. F. Prokasy (Eds.), Classical conditioning II: Current research and theory (pp. 64-99). New York: Appelton-Century-Crofts.

Rizl.ey, R. C., \& Rescorla, R. A. (1972). Associations in secondorder conditioning and sensory preconditioning. Journal of Comparative \& Physiological Psychology, 81, 1-11.

Schreurs, B. G., \& WestBroOK, R. F. (1982). The effect of changes in the CS-US interval during compound conditioning upon an otherwise blocked element. Quarterly Journal of Experimental Psychology, 34B, 19-30.

Smith, M. C., Coleman, S. R., \& Gormezano, I. (1969). Classical conditioning of the rabbit's nictitating membrane response at backward, simultaneous, and forward CS-US intervals. Journal of Comparative \& Physiological Psychology, 69, 226-231.

Tulv:NG, E., \& MADIGAN, S. A. (1970). Memory and verbal learning. Annual Review of Psychology, 21, 437-484.

Yin, H., Barnet, R. C., \& Miller, R. R. (1994). Second-order conditioning and Pavlovian conditioned inhibition: Operational similarities and differences. Journal of Experimental Psychology: Animal Behavior Processes, 20, 419-428.

\section{NOTES}

1. The bottom portion of Figure 2 suggests that a memory representation of CSI was part of the associative structure that underlies responding to CS2. This of course does not need to be the case. The role of CS1 is clearly important during the development of second-order conditioning, but, once second-order conditioning has been established, memory for CSI does not necessarily continue to be critical in the elicitation of responding by the second-order CS2. Whether or not memory for CSI was part of the associative structure underlying behavior to CS2 is not central here. Relevant discussions of the role of memory for the first-order cue in second-order conditioning are provided by Barnet et al. (1991) and Rizley and Rescorla (1972).

2 . The viability of this inhibition interpretation, however, is challenged by the small number (four) of CS2 $\rightarrow$ CS1 trials during Phase 2 . Several recent reports have indicated that many such pairings are necessary to establish CS2 as a conditioned inhibitor (e.g., Yin, Barnet, \& Miller, 1994).

3. Although the temporal coding hypothesis distinguishes itself from the other mentioned interpretations of the current data by being able to explain the central outcomes of both Experiment 1 and Experiment 2, there is one aspect of the data that might be viewed as enigmatic within the framework of the hypothesis. Specifically, during testing, CSs were presented for as long as $10 \mathrm{~min}$, whereas in training the CS durations were $5 \mathrm{sec}$. If, as the temporal coding hypothesis posits, subjects learn temporal relationships in Pavlovian training, why did subjects continue to exhibit anticipatory conditioned suppression beyond the first $5 \mathrm{sec}$ of the CSs testing? Subjects should have expected the US as the end of the first $5 \mathrm{sec}$ of the test CS and presumably not thereafter. Although we do not have a compelling solution to this enigma, one possibility is that CS onset (as opposed to the continued presence of the CS or CS offset) is the major eliciting stimulus. Presumably, once the autonomic arousal of fear has been induced, it lasts for several minutes. This would account for the prolonged suppression observed with the present testing procedures.

(Manuscript received June 29, 1993; revision accepted for publication August 30, 1994.) 